\title{
Thinning of Emulsion Water-in-Oil Films Stabilized with Modified Aluminum Hydroxide under Influence of Applied Pressure Drop
}

\author{
Alla V. Nushtaeva \\ Department of Physics and Chemistry, Penza State University of Architecture and Building, Penza, Russia \\ Email: nushtaeva.alla@yandex.ru
}

Received 27 December 2013; revised 19 January 2014; accepted 27 February 2014

Copyright (C) 2014 by author and Scientific Research Publishing Inc. This work is licensed under the Creative Commons Attribution International License (CC BY). http://creativecommons.org/licenses/by/4.0/

cC) (i) Open Access

\begin{abstract}
The results of studies of thinning free water-in-oil emulsion films stabilized by solid particles by the Applied Pressure Drop Technique (APDT) are reported. The quasi-equilibrium film thickness achieved by slow increasing the pressure drop was smaller than the rupture thickness obtained by sharp increasing the pressure. It is shown that non-equilibrium films ruptured with thicknesses corresponding to adjustment of the packing of solid particles in the films. It is supposed that the restructuring package is the cause of the earlier breakthrough of the film.
\end{abstract}

\section{Keywords}

Solid Emulsifier; Emulsions; Films; Pressure Drop Technique

\section{Introduction}

Finely dispersed powder particles insoluble in either water or in oil, are capable to stabilize emulsions and foams. Such powders are called solid stabilizers.

Emulsions stabilized by solid particles have unusual properties in contrast to emulsions stabilized with traditional surfactants: 1) they are an extremely stable against coalescence, and 2) they exhibit unusual rheological behavior [1]-[3]. In this regard, an emulsion stabilized with solid particles can be used to develop new materials, such as aerogels or macroporous solid foam [1].

Emulsion stability (regardless of the type of emulsifier) is determined, on the one hand, by the resistance interfacial (adsorbed) layer of the emulsifier on the surface of the droplets and, on the other hand, by the resistance 
emulsion [2] [4]-[7].

In real systems, emulsion film is a liquid interlayer of the continuous phase between the droplets of the dispersed phase. The films produced outside the emulsions are called model (and also free or isolated).

There are various theoretical models of the structure of the emulsion films stabilized by solid particles [8] [9]: 1) monolayer film comprising a single layer of the particles bridged the liquid interlayer [10] [11]; 2) bilayer film comprising two interfacial layers of the particles [7] [12] [13]; 3) multilayer film in which the particles form a network-structure connecting two interfacial layers [8].

Type of the film is determined by the structure of the interfacial layer and hence depends on the particle concentration and value of the contact angle $\theta$ (the selective wetting angle of the solid particles with water and oil).

Bilayer film can formed at a sufficiently high concentration of the particles and the optimum value of the angle $\theta \approx 60^{\circ}-85^{\circ}$ (for direct emulsion, $\mathrm{O} / \mathrm{W}$ ) and $95^{\circ}-120^{\circ}$ (for inverse emulsions, W/O), when the drops are covered with a dense monolayer of the solid particles [8] [10] [12] [13].

Figure 1 illustrates the formation of a bilayer film. At the collision of the emulsion droplets, during the first stage, the film consisting of two layers of the interfacial layer and liquid medium between them is formed (Figure 1(a)). The outflow of the continuous phase (medium) causes a thinning of the film up to the contact of the interfacial layers of particles. So the bilayer film with mixed packing (cubic between the layers, but hexagonal inside of the layers) is formed (Figure 1(b)). The next step of thinning is packing seal between the opposing layers which is possible with a tangential displacement of the layers relative to each other, i.e. a rearrangement of the particles inside the film occurs (Figure 1(c)). Moreover, within the interfacial layers, the particle packing type is always the most likely dense hexagonal.

Regardless of the model further thinning of the film leads to a bending of the water/oil interface in the pore space between the particles (it is indicated by arrow in Figure 1). Consequently, the capillary pressure $P_{\sigma}$ осcurs in the film [12] that prevents its further thinning.

This work presents the results of investigations of free emulsion films of water-in-oil by Applied Pressure Drop Technique (APDT) [14], which allows to overcome the capillary pressure arises and get more thin films.

\section{Materials and Methods}

\subsection{Materials}

The particles of aluminum hydroxide were used as the solid emulsifier. The surface of aluminum hydroxide was modified by stearic acid.

A dispersion of $\mathrm{Al}(\mathrm{OH})_{3}$ was prepared by leaching solution $\mathrm{AlCl}_{3}$ when $\mathrm{NaOH}$ was added. Solution of $\mathrm{AlCl}_{3}$ has an acidic reaction, and adding an alkali to a $\mathrm{pH}>5$ aluminum hydroxide sol formed. The point of zero charge of the particles $\mathrm{Al}(\mathrm{OH})_{3}$ is equal to $\mathrm{pH}=6.7$ [15].

Modification of the particles by stearic acid (HSt) was carried out directly on the water/oil interface: acid molecules HSt chemically adsorbed on the surface of $\mathrm{Al}(\mathrm{OH})_{3}$ to form aluminum stearate (AlSt) on the particle surface.

In this paper AlSt prepared at a concentration of aluminum chloride $C_{\mathrm{AlCl}_{3}}=0.01 \%, \mathrm{pH}=7.5$ in the water and the concentration of stearic acid $C_{\mathrm{HSt}}=0.001 \%$ in the oil. To increase electrical conductivity of the aqueous phase potassium chloride was added to $C_{\mathrm{KCl}}=0.1 \mathrm{~mol} / \mathrm{L}$ (specific conductivity is equal to $\chi_{\mathrm{sp}}^{25^{\circ}}=128.8 \times 10^{-4}$

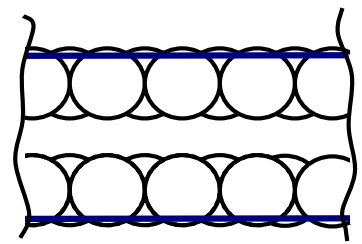

(a)

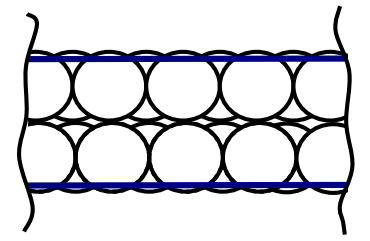

(b)

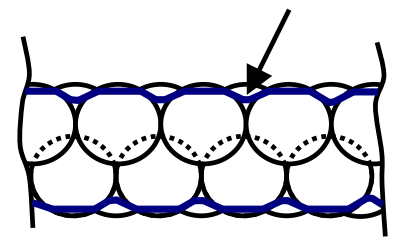

(c)

Figure 1. Stages of thinning film stabilized with solids: (a) thick film with an interlayer of the continuous phase; (b) a mixed bilayer film (cubic package between the interfacial layers); and (c) a bilayer film with hexagonal close packing (arrow indicates the meniscus of water/oil in the pore space between the particles). 
$\left.\mathrm{Om}^{-1} \cdot \mathrm{cm}^{-1}\right)$

Mixture of octane and carbon tetrachloride (in volume ratio 1:1) was used as the oil phase. The density of a mixture of oils was equal to $\rho=1.14 \mathrm{~g} / \mathrm{cm}$.

\subsection{Methods}

Emulsions from aqueous dispersion of $\mathrm{Al}(\mathrm{OH})_{3}$ and oil solution of stearic acid of various concentration was formed by the method of shaking in a test tube at adding oil drop by drop to water (for emulsion $\mathrm{O} / \mathrm{W}$ ) or at adding water drop by drop to oil (for emulsion W/O) up to volume ratio 1:1.

Adsorbed layer of the solid particles was formed simultaneously with the modifying process. Into a weighing bottle the solution of stearic acid in the oil was poured and then the aqueous dispersion $\mathrm{Al}(\mathrm{OH})_{3}$ carefully poured on the oil. Solids spontaneously fixed on the surface of the water/oil forming the interfacial layer.

The free emulsion films were obtained by moving of a measuring cell from the aqueous phase into the oil phase. The measuring cell was made from a porous glass plate with the pore diameter of $16 \mu \mathrm{m}$ (POR 16). The hole with diameter of $6.8 \mathrm{~mm}$ was made in the plate to the film forming.

Scheme of the measurement setup by APDT-method is shown in Figure 2. The cell (1) connected to the system consisting of tubes and funnel (2) with water. In slow (drop by drop) leaking of water from the funnel the low pressure $P_{1}$ (compared to the atmospheric pressure $P_{0}$ ) arose in the air system. The applied pressure drop was equal to:

$$
\Delta P=P_{0}-P_{1} .
$$

The pressure drop $\Delta P$ causes the thinning of the film up to equilibrium thickness $h_{e}$ or to rupture thickness $h_{r}$.

The film was penetrated two electrodes: 1) the external electrode (5) is a thin platinum plate attached to the upper part of the hole, and hence communicates with an external meniscus, 2) the internal electrode (6) is platinum wire, similarly penetrating center of the film. The radii of the electrodes (including the menisci) $r_{2}=3.2 \pm$ $0.2 \mathrm{~mm}$ and $r_{1}=0.28 \pm 0.05 \mathrm{~mm}$ were measured using a cathetometer.

The thickness of water film was determined by the conductometric method.

At the moment of the cell moving the film consists of two interfacial layers with a layer of an aqueous me-

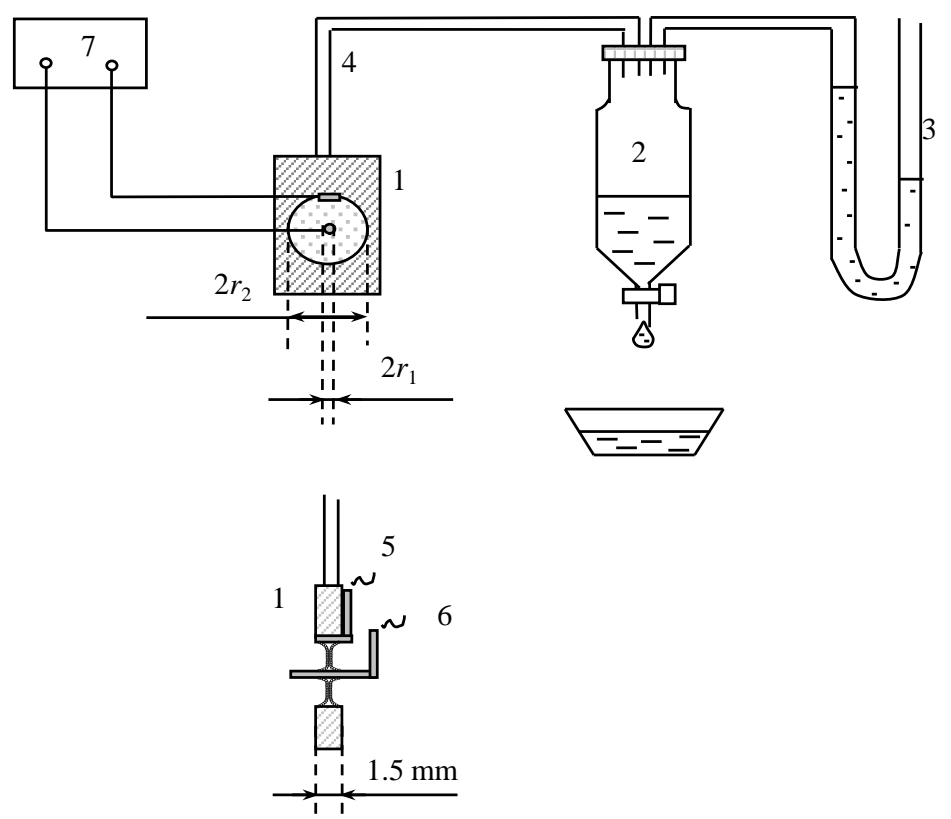

(a)

(b)

Figure 2. Scheme of the setup for investigation of the film thinning by APDTmethod (a) and profile view of the measuring cell with the film in the hole (b): (1) the measuring cell was made from a porous glass plate; (2) funnel; (3) U-shaped water manometer; (4) glass tubes; (5) the external electrode; (6) the internal electrode; (7) conductometer. 
dium between them and it is thinning up to the contact of the interfacial layers. Thus the conductivity of the film decreases and reaches equilibrium value $\chi_{f(e)}$ (reaches a plateau). The equilibrium value of conductivity corresponds to the equilibrium thickness of the film $h_{e}$, which is equal to:

$$
h_{e}=\frac{\chi_{f(e)} \ln \left(r_{2} / r_{1}\right) n B}{2 \pi \chi_{\mathrm{sp}}},
$$

were $\chi_{\mathrm{sp}}$ is specific conductivity of the aqueous phase, $n$ is the calculated multiplicity of the film [9], and $B$ is the empirical coefficient of liquid distribution between solid particles in the film [9].

As value of $n$ is determined by the particles packing type and number of layers of the particles in the film so the thickness $h$ calculated out of the conductivity depends on assumed model of the film. In these work, it is assumed the film to be a bilayer. For that model $n B$ factor varies from 2.78 (for contact angle $\theta=0^{\circ}-16^{\circ}$ ) up to 5.06 (for $\theta=88^{\circ}-89^{\circ}$ ).

In these work, $n B$ factor is assumed to be equal to 3.5 for the angle $\theta=43^{\circ}$ [4] (for concentration ratio $C_{\mathrm{HST}} / C_{\mathrm{AlCl}_{3}}=0.1$ ).

If it will be suggested a multilayer film then $n B$ factor will be equal to 2.78 - 3.12 (on depend on density of the packing type between the interfacial layers) [16].

Non-equilibrium thickness of the thinning film consisting of two layers of interfacial layer and aqueous medium is equal to:

$$
\begin{gathered}
h=h_{w}+2 h_{s}, \\
h_{s}=R(1+\cos \theta),
\end{gathered}
$$

were $h_{w}$ is thickness of aqueous medium determined from the difference $\left(\chi_{f}-\chi_{f(e)}\right)$ at $n B=1, h_{s}$ is thickness of interfacial layer of the particles, $R$ is radius of the single particle (or the particles aggregate), $\theta$ is selective contact angle for solids on the oil/water interface. In the case of bilayer film, it will be $2 h_{s}=h_{e}$.

To determine thickness of non-equilibrium films a calibration curve $\chi_{f}(h)$ was constructed through Equation (3).

\section{Results and Discussions}

The emulsions $\mathrm{O} / \mathrm{W}$ were obtained at concentration ratio $C_{\mathrm{HST}} / C_{\mathrm{AlCl}_{3}} \leq 1-1.5$ and the emulsions W/O-at ratio $C_{\mathrm{HST}} / C_{\mathrm{AlCl}_{3}} \geq 0.2$. So at ratio $C_{\mathrm{HST}} / C_{\mathrm{AlCl}_{3}}$ in the range of $0.2-1$ emulsions of both types could be prepared.

Stearic acid itself does not stabilize the emulsion in the absence of solid particles. Dispersion of $\mathrm{Al}(\mathrm{OH})_{3}$ without stearic acid gives a stable emulsion $\mathrm{O} / \mathrm{W}$.

The lifetime of free macroscopic water-in oil films ranged from a few minutes to an hour (in the field of direct emulsions). The films were completely coated particles, particle-free areas (which gives interference) is not observed.

In addition to the optimal composition of the emulsifier it was necessary to satisfy the additional conditions: 1) direct contact of interfacial layers must occur immediately at the cell diving (if a meniscus occur then the leakage of water led to the particles "trickled down" on the interface from center to periphery), and 2) the internal phase of the film should not form a gel (otherwise compression of gel and delaminating from the edges of the hole occur at applying pressure $\Delta P$ ).

In the first series of experiments, various pressure $\Delta P$ applied to a single film. A change in electrical conductivity was observed for a few minutes and then pressure $\Delta P$ raised. Maximum pressure $\Delta P$ was equal to $5 \mathrm{kPa}$. The results of these measurements are shown in Figure 3. Reducing conductivity related to thinning of the film. At low values of $\Delta P$ the film was thinning for $1-5$ min until an equilibrium thickness $h_{e}$, which was equal to 29.8 microns (for $\Delta P=1 \mathrm{kPa}$ ), 28.4 microns ( $\Delta P=2 \mathrm{kPa}$ ), 26 microns ( $\Delta P=3 \mathrm{kPa}$ ) and 24.2 microns $(\triangle P=4 \mathrm{kPa})$.

Moreover, since $\Delta P=2 \mathrm{kPa}$, stepwise refinement is noticeable in Figure 3. It can be assumed that it is due 


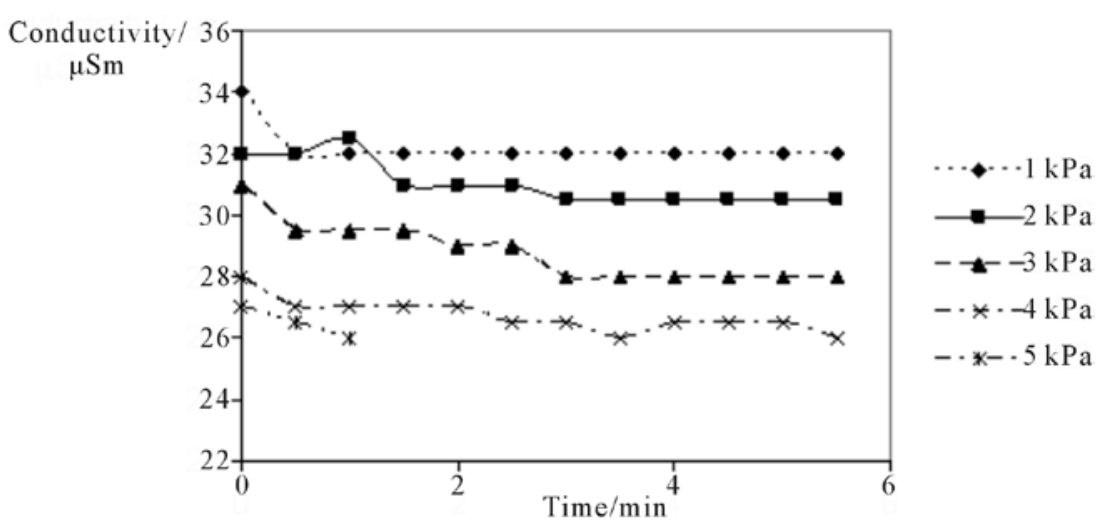

Figure 3. Decreasing in conductivity of the emulsion water-in-oil film due to thinning under the influence of applied pressure drop $\Delta P$ (from 1 to $5 \mathrm{kPa}$ ).

to compaction of the particles in the interfacial layers.

It should be noted some abnormal increasing in the thickness in curve 2 observed during nearly 15 sec. Then the thinning process was resumed soon as it is seen in Figure 3. Such jumps of conductivity (and thus thickness) were observed during any mechanical influence (random jolting, vibration). Probably it was irregular local thickening of some areas of the film caused by undulating vibration of the film. This indicates that the "dried" solid-stabilized film can be restored by absorbing water from meniscus.

At a pressure of $\Delta P=5 \mathrm{kPa}$ the film was broke already after $1 \mathrm{~min}$. Equilibrium thickness was not achieved. It should be emphasized that the pore diameter of the cell (16 microns) created a pressure drop of not more than $5 \mathrm{kPa}$.

The same film without pressure drop just under the influence of gravity was thinning to thickness $h_{e}=32$ microns for 30 minutes. From the equilibrium thickness of a film (without pressure drop) effective particle radius $R_{e f}$ expressed from Equation (4) can be calculated in assuming a bilayer model. Thus from the thickness $h_{e}=32 \mu \mathrm{m}$ and the angle $\theta_{w}=43^{\circ}$ we have got radius value $R_{e f}=9.4$ microns. Observation of particles directly on the water/oil interface with a microscope showed that most of the particles have radii in the range 3 - 7 microns, maximum 20 - 30 microns. Thus, the value $R_{e f}$ corresponded to real area of the particle size distribution.

Although bilayer film is just a model, but it is in good agreement with the experimental data. Thus, for example, the effective radii $R_{e f}$, calculated from $h_{e}$ of films made from particles of silica, and radii $R$, found by sedimentation analysis, had the same values [3].

In a second series of experiments at a constant pressure $\Delta P$ the film conductivity was measured continuously until the break of the film. Time of thinning reduced from $35-60$ minutes (for $\Delta P=0-1 \mathrm{kPa}$ ) to about 1 - 5 minutes (for $\Delta P=3-5 \mathrm{kPa}$ ). Figure 4 shows the thickness of rupture of the films $h_{r}$ and theoretical isotherms of capillary pressure $P_{\sigma}$ in the film for two types of packing of particles of opposite interfacial layers.

Comparison of the results of first and second series of measurements (Table 1) shows that the thickness $h_{e}$, obtained with a gradual increase $\Delta P$ (and hence slow thinning), in all cases less than the value of $h_{r}$, achieved by rapid thinning of the films.

Furthermore, as seen from Figure 4, the film ruptured with thicknesses corresponding adjustment of the package from mixed to hexagonal close packing. In this regard, it can be assumed that increasing the capillary pressure in the film leads to compaction of the particles in the film as between opposite interfacial layers so within the layers themselves. Thus restructuring of particles within the film causes mechanical perturbations within it that leads to earlier breakthrough of the film.

\section{Conclusion}

Method APDT at certain conditions is applicable to films stabilized by solids. With a gradual increase of pressure drop achieved quasi-equilibrium film thickness were smaller than the rupture thickness, resulting in a sharp increase of the pressure. Non-equilibrium film broke with thicknesses corresponding adjustment of the packing 


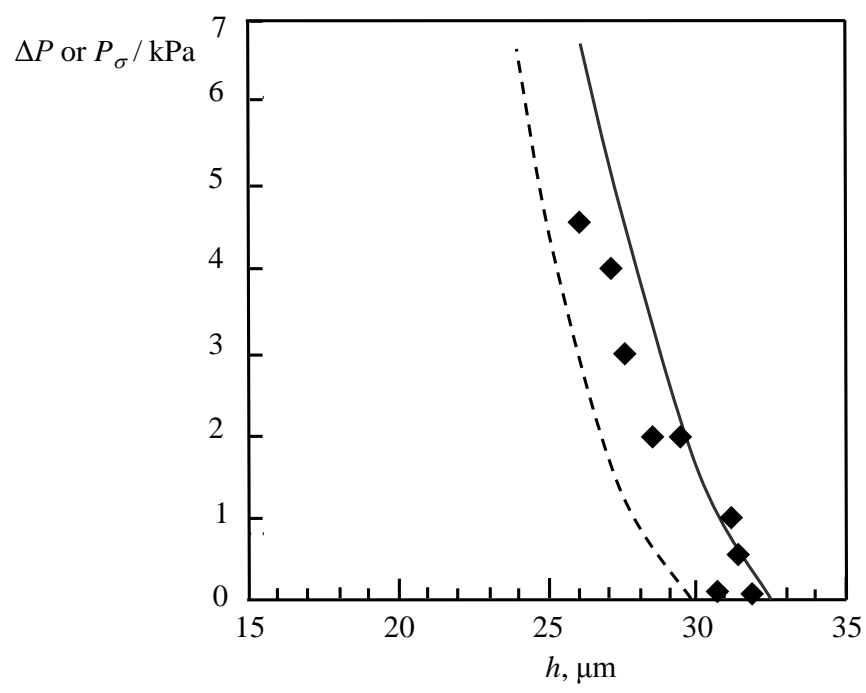

Figure 4. Rupture thickness $h_{r}$ of emulsion films at the constant applied pressure drop $\Delta P$ (dark diamonds) and isotherms of capillary pressure $h\left(P_{\sigma}\right)$ for hexagonal close packing of the particles in the film (solid curve) and for mixed packing type (dashed curve).

Table 1. Equilibrium film thickness $h_{e}$ and film rupture thickness $h_{r}$ reached at the pressure drop $\Delta P$.

\begin{tabular}{cccccc}
\hline$\Delta P, \mathrm{kPa}$ & 1 & 2 & 3 & 4 & 4.5 \\
\hline$h_{e}, \mu \mathrm{m}$ & 29.8 & 28.4 & 26 & 24.2 & - \\
$h_{r}, \mu \mathrm{m}$ & 31.5 & $28.5-29.5$ & 28 & 27.5 & 26.5 \\
\hline
\end{tabular}

of solid particles in the film, which is apparently the cause of the earlier breakthrough of the film and failure to reach a quasi-equilibrium film thickness.

\section{References}

[1] Ardity, S., Schmitt, V., Giermanska-Kahn, J. and Leal-calderon, F. (2004) Materials Based on Solid-Stabilized Emulsions. Journal of Colloid Interface Science, 275, 659-664. http://dx.doi.org/10.1016/j.jcis.2004.03.001

[2] Tcholakova, S., Denkov, N.D. and Lips, A. (2008) Comparison of Solid Particle, Globular Proteins and Surfactants as Emulsifiers. Physical Chemistry Chemical Physics, 10, 1608-1627. http://dx.doi.org/10.1039/b715933c

[3] Nushtaeva, A.V., Shumkina, A.A., Kruglyakov, P.M. and Elaneva, S.I. (2011) Effect of Aqueous Phase Structuring on the Properties of Model Emulsion Films Stabilized with Solid Microsized Particles. Colloid Journal, 73, 821-829.

[4] Kruglyakov, P.M. (2000) Hydrophile-Lipophile Balance of Surfactants and Solid Particles. Physicochemical Aspects and Applications. Elsevier, Amsterdam.

[5] Aveyard, R., Binks, B.P. and Clint, J.H. (2003) Emulsions Stabilized Solely by Colloidal Particles. Advances in Colloid and Interface Science, 100-102, 503-546. http://dx.doi.org/10.1016/S0001-8686(02)00069-6

[6] Kruglyakov, P.M. and Nushtaeva, A.V. (2004) Emulsion Stabilized by Solid Particles: Influence of the Capillary Pressure. In: Petsev, D.N., Ed., Emulsions: Structure, Stability and Interactions, Elsevier, Amsterdam, 641-676. http://dx.doi.org/10.1016/S1573-4285(04)80018-8

[7] Kaptey, G. (2006) On the Equation of the Maximum Capillary Pressure Induced by Solid Particles to Stabilize Emulsions and Foams and on the Emulsion Stability Diagrams. Colloids and Surfaces A: Physicochemical and Engineering Aspects, 282-283, 387-401. http://dx.doi.org/10.1016/j.colsurfa.2005.12.021

[8] Horosov, S. (2008) Foams and Foam Films Stabilised by Solid Particles. Current Opinion in Colloid and Interface Science, 13, 134-140.

[9] Nushtaeva, A.V., Vilkova, N.G. and Elaneva, S.I. (2011) Stabilization of Foams and Emulsions by Insoluble Pouder. PGUAS, Penza.

[10] Denkov, N.D., Kralchevsky, P.A. and Ivanov, I.B. (1997) Lateral Capillary Forces and Two-Dimentional Arrays of 
Colloid Particles and Protein Molecules. Journal of Dispersion Science and Technology, 18, 577-591.

[11] Velikov, K.P., Durst, F. and Velev, O.D. (1998) Direct Observation of the Dynamics of Latex Particles Confined inside Thinning Water-Air Films. Langmuir, 14, 1148-1155.

[12] Nushtaeva, A.V. and Kruglyakov, P.M. (2003) The Capillary Pressure in the Thinning Emulsion Films Stabilized with Solid Spherical Particles. Colloid Journal, 65, 374-382.

[13] Alargova, R.G., Warhadpande, D.S., Paunov, V.N. and Velev, O.D. (2004) Foam Superstabilization by Polymer Microrods. Langmuir, 20, 10371-10374. http://dx.doi.org/10.1021/la048647a

[14] Kruglyakov, P.M. and Exerova, D. (1998) Foam and Foam Films. Elsevier, Amsterdam.

[15] Kosmulski, M. (2002) The pH-Dependent Surface Charging and the Point of Zero Charge. Journal of Colloid Interface Science, 253, 77-87. http://dx.doi.org/10.1006/jcis.2002.8490

[16] Nushtaeva, A.V., Shumkina, A.A. and Volkova, N.V. (2012) Multilayer Film Model Stabilized by Solid Particles. Izvestiya PGPU im. V.G. Belinskogo, 352-357. 